\title{
STATISTICAL DISTRIBUTION FUNCTION OF CHARGED PARTICLES IN MAGNETIC FIELD
}

\author{
B. CHETVERUSHKIN*, N. D'ASCENZO**†, A.SAVELIEV ${ }^{* * \dagger \dagger}$ \\ AND V. SAVELIEV ${ }^{* * \dagger}$ \\ * Keldysh Institute of Applied Mathematics \\ Russian Academy of Science (KIAM RAS) \\ 4 Miusskaya sq, 125047 Moscow, Russia \\ e-mail: chetver@imamod.ru,web page: http://www.kiam.ru \\ ${ }^{\dagger}$ Deutsche Electronen Synchrotron (DESY) \\ 85 Notkestrasse, 22607 Hamburg, Germany \\ e-mail: ndasc@mail.desy.de - Web page: http://www.desy.de \\ ${ }^{\dagger \dagger}$ Hamburg University \\ 85 Notkestrasse, 22607 Hamburg, Germany \\ e-mail: Andrey.Saveliev@desy.de - Web page: http://www.desy.de \\ ** Immanuel Kant Baltic Federal University \\ 14 Alexandr Nevsky str., 236016, Kaliningrad, Russia \\ e-mail: saveliev@mail.desy.de - Web page http://www.kantiana.ru
}

Key words: MGD pump, numerical methods, kinetic consistent algorithms

\begin{abstract}
The statistical distribution function introduced by Boltzmann and his kinetic equation are the fundamental basis of the kinetic theory of gases and of the basic methods of solution of problems in the gas dynamics. At present time one of the areas of high interest in modern physics is the plasma in fusion processes and astrophysics which requires an extension of the kinetic processes to charged particles, in particular regarding the electromagnetic interactions. We propose a unified distribution function which includes the electromagnetic interactions for charged particles and is suitable for the solution of problems of charged particle dynamics with Boltzmann type equations and kinetic consistent magneto gas dynamic equations.
\end{abstract}

\section{INTRODUCTION}

The Boltzmann equation is the fundamental basis of the kinetic theory of gases [1]. The distribution function and the kinetic equations were introduced by Boltzmann for the 
accurate description of the microscopic properties and the dynamics of gases. The macroscopic properties of gases can be extracted as the moments of the distribution function. Despite the known difficulties in the solution of the Boltzmann equation modern developments are going to explore its solution on powerful high performance computing systems by numerical methods. Two modern examples are Lattice Boltzmann Schemes (LBS), which use the evolution of the distribution function and calculate the macroscopic gas dynamic parameters as moments of the distribution function [2] and Kinetically Consistent Difference Schemes (KCDS), which derive the gas dynamic equation from the Boltzmann equation by integrating the velocity space with the summational invariants [3]. The resulting equations gives a more reach physical models of the dynamics of gases in comparison to the gas dynamic equations, as the Navie-Stokes equations, which are formulated phenomenologically [4].

Many modern important problems require not only to study the dynamics of neutral particles, as a gas, but also to deal with the dynamics of charged particles, as clouds of electrons, or charged interstellar matter in astrophysics or ionized gas in strong electromagnetic fields as in plasma physics processes. In order to solve these problems one has to take into account the electromagnetic interactions of the charged particles or charged media.

The study of the relations between the statistical gas dynamics and the electromagnetic laws goes in the direction of trying to combine directly the kinetic equations of the charged particles with the electromagnetic equations. One approach was done by Vlasov, who introduced the electromagnetic forces in the kinetic equation - Boltzmann - Vlasov equations. In this approach electromagnetic interactions are introduced phenomenologically as an external Lorentz force [5] in the momentum equation. Few later attempts to implement the electromagnetic terms directly in the distribution function faced the difficulty with the axial vector behavior of the magnetic interactions $[6,7,8]$.

We propose a new approach to define an unified distribution function, which includes the electromagnetic terms in the distribution function for the solution of the magneto gas dynamic problem by BLS or KCDS for charged particles in an electromagnetic field.

The main goal of this paper is to formulate the statistical distribution function with included electromagnetic terms and prove at the first step the validity of the proposed distribution function by the reconstruction of the kinetic consistent magneto gas dynamic equations including the equation of the evolution of the magnetic field in the ideal case for charged particles.

\section{THEORETICAL ISSUES}

The goal of the present study is the definition of the united distribution function describing the dynamics of the charged particles (electron cloud, ionized gas), including conditions with the external electromagnetic field. 


\subsection{Distribution Function of Gas Dynamic Processes}

The kinetic theory describes the gas dynamics by the Boltzmann differential equation through the evolution of the distribution function $f(\mathbf{x}, \boldsymbol{\xi}, t)[1]$ :

$$
\frac{\partial f(\mathbf{x}, \boldsymbol{\xi}, t)}{\partial t}+\boldsymbol{\xi} \cdot \nabla f(\mathbf{x}, \boldsymbol{\xi}, t)=C(f),
$$

where: $C(f)$ is a nonlinear integral operator which

describes the collisions of particles.

The computational interest in the kinetic formulations of gas dynamics is high due to the linearity of the differential operator on the left side of Eq. (1). The nonlinearity is confined by the collision term, which is generally local in $\mathbf{x}$ and $t$.

Another important feature for numerical methods is that the collision integral vanishes in the equilibrium state when the local Boltzmann distribution function is Maxwellian:

$$
f_{M}=\frac{\rho m^{1 / 2}}{(2 \pi k T)^{3 / 2}} \exp \left\{-\frac{m}{2 k T}(\boldsymbol{\xi}-\mathbf{u})^{2}\right\}
$$

The dynamic of the gas could be defined as the time evolution of the Maxwellian distribution function with the conditions that the collision processes is occurred during a characteristic time at the end of which the distribution function became Maxwellian [9]. The macroscopic observables such as density, momentum, energy flux as a function of $\mathbf{x}$ and $t$ are defined from the moments of the distribution function with respect to the particle velocity.

Earlier investigations [11] show that the electromagnetic fields do not destroy the validity of the distribution function and Boltzmann equation and open the way to the implementation of the electromagnetic processes terms in the distribution function.

\section{$2.2 \quad$ Electromagnetic Interactions}

From the kinetic relations, in addition to the gas kinetic motions, the motions of the charged particles is defined through nature of the electric and magnetic fields and the forces acting on the charged particles.

The electric field has the polar vector behavior and the corresponding velocity of charged particles follows the direction of the electric field and can be considered as simple drift of the charged particles in the direction of the electric field.

The magnetic field has the axial vector behaviour and acts in a different way respect to other fields. As well known the motions of the charged particles in magnetic field affects only the direction of the velocity and the motion of the charged particle has a circular trajectory in the plane perpendicular to the magnetic field. The theory of complex variables is ideally suited to solve problems involving two dimensional circulation and in 
many ways complex variable theory is simpler than real variable theory and much more powerful [12].

As an example let's consider the motions of the charged particle in the uniform magnetic field [13]. The magnetic filed is directed along the $z$ - axis. The equation of the motions is:

$$
\frac{d}{d t} \mathbf{p}=\frac{e}{c} \mathbf{v} \times \mathbf{B}
$$

can be rewritten in term of momentum:

$$
\mathbf{p}=\frac{E \mathbf{v}}{c^{2}}
$$

where $E$ is the energy of particle, which in the magnetic field is constant.

$$
\frac{E}{c^{2}} \frac{d \mathbf{v}}{d t}=\frac{e}{c} \mathbf{v} \times \mathbf{B}
$$

Or in the velocity components:

$$
\frac{d}{d t} v_{x}=\omega v_{y}, \frac{d}{d t} v_{y}=-\omega_{x}, \frac{d}{d t} v_{z}=0
$$

where: $\omega=\frac{e c \mathbf{B}}{E}$

Multiplying first eq. of 6 on $i$ and summing with second eq. of 6

$$
\frac{d}{d t}\left(v_{x}+i v_{y}\right)=-i \omega\left(v_{x}+v_{y}\right)
$$

from which:

$$
v_{x}+i v_{y}=v_{0 t} e^{i(\omega t+\alpha)}
$$

where $v_{0 t}$ and $\alpha$ are real.

Select real and imaginary parts:

$$
v_{x}=v_{0 t} \cos (\omega t+\alpha), \quad v_{y}=-v_{0 t} \sin (\omega t+\alpha)
$$

where the $v_{0 t}=\sqrt{v_{x}^{2}+v_{y}^{2}}$ and $\alpha$ defined by the initial conditions.

This relation means that $v_{0 t}$ is the velocity of the charged particle in plane $x y$, constant during the motions in the magnetic field. 


\subsection{Proposed Distribution Function}

Following the above considerations the velocity as result of the electromagnetic interaction can be defined as a complex velocity involving the electric and magnetic terms:

$$
\mathbf{v}_{e m} \propto \alpha_{1} \mathbf{E}+i \alpha_{2} \mathbf{B}
$$

The important results of this considerations are that the electric field term of the velocity could be consider as contribution to the drift velocity and the magnetic term in the conditions when the Larmor radius is smaller in comparison to the total volume is part of the statistical velocity term.

In order to evaluate the distribution function at the equilibrium state, we refer to the Boltzmann H-theorem [14].

We consider the charged particles as spherical particles. The charged particles gas state is uniform and the velocity distribution function $f$ is independent of $x, y, z$. The Boltzmann equation reduces to:

$$
\frac{\partial f}{\partial t}=\iint\left(f f^{1}-f^{\prime} f^{1^{\prime}}\right) v_{r e l} d \sigma d \mathbf{p}^{1}
$$

where:

$v_{\text {rel }}=\left|\mathbf{v}-\mathbf{v}^{1}\right|$ is the relative initial velocity

between the two colliding particles,

$d \sigma$ is the differential collision cross section,

$d \mathbf{p}^{1}$ is the momentum of the second particle.

The ' symbol indicates the final states after the collision.

According to the Boltzmann $\mathrm{H}$-theorem the distribution function at the equilibrium should minimize the integral quantity $H$ :

$$
H=\int f \log f d \mathbf{c}
$$

Combining Eq. 11 and 12 we obtain:

$$
\frac{\partial H}{\partial t}=\int \frac{\partial}{\partial t}(f \log f) d \mathbf{c}=\int(1+\log f) \frac{\partial f}{\partial t} d \mathbf{c}
$$

from which:

$$
\frac{\partial H}{\partial t}=\frac{1}{4} \iiint \log \left(\frac{f f^{1}}{f^{\prime} f^{1^{\prime}}}\right)\left(f f^{1}-f^{\prime} f^{1^{\prime}}\right) v_{r e l} d \sigma d \mathbf{p}_{1} d \mathbf{c}
$$

At the equilibrium, according to the H-theorem:

$$
\frac{\partial H}{\partial t}=0
$$


from which the relation is obtained:

$$
\log f_{0}+\log f_{0}^{1}=\log f_{0}^{\prime}+\log f_{0}^{1^{\prime}}
$$

This relation shows that $\log f$ is an invariant of the collision and can be expressed as the sum of the kinetic invariants:

$$
\log f_{0}=\beta_{1}+\beta_{2} \cdot m \boldsymbol{\xi}+\beta_{B} \cdot m \boldsymbol{\xi}-\beta_{3} \frac{m}{2} \xi^{2}
$$

where:

$\beta_{1}$ is a real number, scalar related to the mass,

$\beta_{\mathbf{2}}$ is a real vector, related to the total momentum

including magnetic terms,

$\beta_{\mathbf{B}}$ is a complex vector, related to the magnetic

field,

$\beta_{3}$ is a real number, scalar related to the energy

It is convenient to recast the equation 17 in the form:

$$
\log f_{0}=\log \beta_{0}-\frac{m}{2} \beta_{3}\left(\boldsymbol{\xi}-\frac{\boldsymbol{\beta}_{2}}{\beta_{3}}-\frac{\boldsymbol{\beta}_{B}}{\beta_{3}}\right)^{2}
$$

with $\beta_{0}$ real number, scalar recasting $\beta_{1}$ and $\beta_{3}$.

We require that the distribution function takes only real values in order to preserve its meaning as distribution:

$$
\begin{aligned}
& \Im \log f_{0}=0 \\
& \Re\left(\boldsymbol{\xi}-\frac{\boldsymbol{\beta}_{\mathbf{2}}}{\beta_{3}}-\frac{\boldsymbol{\beta}_{\boldsymbol{B}}}{\beta_{3}}\right) \cdot \Im\left(\boldsymbol{\xi}-\frac{\boldsymbol{\beta}_{\mathbf{2}}}{\beta_{3}}-\frac{\boldsymbol{\beta}_{\boldsymbol{B}}}{\beta_{3}}\right)=0 \\
& \Re \boldsymbol{\xi} \cdot \Im \boldsymbol{\xi}-\frac{\boldsymbol{\beta}_{2}}{\beta_{3}} \cdot \Im \boldsymbol{\xi}-\Re \boldsymbol{\xi} \cdot \frac{\boldsymbol{\beta}_{B}}{\beta_{3}}+\frac{\boldsymbol{\beta}_{2}}{\beta_{3}} \cdot \frac{\boldsymbol{\beta}_{B}}{\beta_{3}}=0 \\
& \Im \boldsymbol{\xi} \equiv \frac{\boldsymbol{\beta}_{B}}{\beta_{3}}
\end{aligned}
$$

Under this condition the distribution function can be expressed as:

$$
\begin{array}{r}
\log f_{0}=\log \beta_{0}-\frac{m}{2} \beta_{3}\left|\boldsymbol{\xi}-\frac{\boldsymbol{\beta}_{\mathbf{2}}}{\beta_{3}}-\frac{\boldsymbol{\beta}_{\boldsymbol{B}}}{\beta_{3}}\right|^{2} \\
f_{0}=\beta_{0} e^{-\frac{m}{2} \beta_{3}\left|\boldsymbol{\xi}-\frac{\boldsymbol{\beta}_{2}}{\beta_{3}}-i \Im \frac{\boldsymbol{\beta}_{B}}{\beta_{3}}\right|^{2}}
\end{array}
$$

where the module sign indicates explicitly that the complex distribution function $\mathrm{f}$ is real-valued and the $\Im$ symbol in the exponential makes explicit the complex nature of the vector $\boldsymbol{\beta}_{B}$. 
The relations 19 and 21 define the distribution function at the equilibrium. The parameters $\beta_{0}, \beta_{3}, \boldsymbol{\beta}_{2}$ can be obtained from the moments of the distribution function 21 respect to the kinematic invariants with the integrals respecting the prescription 19 . The relation 19 defines the integral in the complex plane along a line $L$ parallel to the real axis and shifted in the direction of the imaginary axis of the quantity $\Im \xi \equiv\left(\frac{\boldsymbol{\beta}_{B}}{\beta_{3}}\right)$.

The real and imaginary parts of the moment of the distribution function respect to the mass $m$ :

$$
\begin{aligned}
& \Re: \int_{L} m f_{0} d^{3} \boldsymbol{\xi}=\rho \\
& \Im: \int_{L} m f_{0} d^{3} \boldsymbol{\xi}=0
\end{aligned}
$$

From the first of the equations 22 the relation is obtained:

$$
\begin{aligned}
& \int_{L} m \beta_{0} e^{-\frac{m}{2} \beta_{3}\left|\xi-\frac{\beta_{2}}{\beta_{3}}\right|^{2}} d^{3} \boldsymbol{\xi} \\
& =\int_{-\infty}^{+\infty} m \beta_{0} e^{-\frac{m}{2} \beta_{3}|c|^{2}} d^{3} c=m \beta_{0}\left(\frac{2 \pi}{m \beta_{3}}\right)^{3 / 2} \\
& m \beta_{0}\left(\frac{2 \pi}{m \beta_{3}}\right)^{3 / 2}=\rho
\end{aligned}
$$

The second of the equations 22 shows that the distribution function is real-valued.

The real and imaginary parts of the moment of the distribution function respect to the momentum $m \boldsymbol{\xi}$ :

$$
\begin{aligned}
& \Re: \int_{L} m \boldsymbol{\xi} f_{0} d^{3} \boldsymbol{\xi}=\rho \mathbf{u} \\
& \Im: \int_{L} m \boldsymbol{\xi} f_{0} d^{3} \boldsymbol{\xi}=\frac{\mathbf{B}}{\sqrt{\mu_{\rho}}}
\end{aligned}
$$

where: equation.

$\mu_{\rho}$ is a constant with dimensions $m^{4} / C^{2}$ needed for dimensional congruence of the

From the first of the equations 24:

$$
\begin{aligned}
& \Re \int_{L} m \boldsymbol{\xi} \beta_{0} e^{-\frac{m}{2} \beta_{3}\left|\boldsymbol{\xi}-\frac{\boldsymbol{\beta}_{2}}{\beta_{3}}-i \Im \frac{\boldsymbol{\beta}_{B}}{\beta_{3}}\right|^{2}} d^{3} \boldsymbol{\xi} \\
& =\Re \int_{-\infty}^{+\infty} m\left(\mathbf{c}+\operatorname{Ree}\left(\frac{\boldsymbol{\beta}_{2}}{\beta_{3}}\right)+i \Im\left(\frac{\boldsymbol{\beta}_{B}}{\beta_{3}}\right)\right) \beta_{0} e^{-\frac{m}{2} \beta_{3}|c|^{2}} d^{3} c \\
& =\rho \Re\left(\frac{\boldsymbol{\beta}_{2}}{\beta_{3}}\right) \\
& \rho \Re\left(\frac{\boldsymbol{\beta}_{2}}{\beta_{3}}\right)=\rho \mathbf{u}
\end{aligned}
$$


From the second of the equations 24:

$$
\begin{aligned}
& \Im \int_{L} m \boldsymbol{\xi} \beta_{0} e^{-\frac{m}{2} \beta_{3}\left|\boldsymbol{\xi}-\frac{\boldsymbol{\beta}_{2}}{\beta_{3}}-i \Im-\frac{\boldsymbol{\beta}_{B}}{\beta_{3}}\right|^{2}} d^{3} \boldsymbol{\xi} \\
& =\Im \int_{-\infty}^{+\infty} m\left(\mathbf{c}+\Re\left(\frac{\beta_{2}}{\beta_{3}}\right)+i m\left(\frac{\beta_{B}}{\beta_{3}}\right)\right) \beta_{0} e^{-\frac{m}{2} \beta_{3}|c|^{2}} d^{3} c \\
& =\rho \operatorname{Im}\left(\frac{\boldsymbol{\beta}_{B}}{\beta_{3}}\right) \\
& \rho \Im\left(\frac{\boldsymbol{\beta}_{B}}{\beta_{3}}\right)=\frac{\mathbf{B}}{\sqrt{\mu_{\rho}}}
\end{aligned}
$$

Finally the real and imaginary parts of the moment of the distribution function respect to the thermal kinetic energy:

$$
\begin{aligned}
& \Re: \int_{L} \frac{m}{2} c^{2} f_{0} d^{3} \boldsymbol{\xi}=\frac{3}{2} \frac{\rho k T}{m} \\
& \Im: \int_{L} \frac{m}{2} c^{2} f_{0} d^{3} \boldsymbol{\xi}=0
\end{aligned}
$$

From the first of the equations 29:

$$
\begin{aligned}
& \Re \int_{L} \frac{m}{2} c^{2} \beta_{0} e^{-\frac{m}{2} \beta_{3}\left|\boldsymbol{\xi}-\frac{\boldsymbol{\beta}_{2}}{\beta_{3}}-i \Im \frac{\boldsymbol{\beta}_{B}}{\beta_{3}}\right|^{2}} d^{3} \boldsymbol{\xi} \\
& =\Re \int_{-\infty}^{+\infty} \frac{m}{2} c^{2} \beta_{0} e^{-\frac{m}{2} \beta_{3}|c|^{2}} d^{3} c \\
& =\frac{3}{2} \frac{\rho}{m \beta_{3}} \\
& \frac{3}{2} \frac{\rho}{m \beta_{3}}=\frac{3}{2} \frac{\rho k T}{m}
\end{aligned}
$$

The relations 23, 26, 28, 31 define the coefficients $\beta_{0}, \boldsymbol{\beta}_{2}, \boldsymbol{\beta}_{B}, \beta_{3}$ :

$$
\begin{aligned}
& \beta_{0}=\rho \frac{m^{1 / 2}}{(2 \pi k T)^{3 / 2}} \\
& \boldsymbol{\beta}_{2}=\mathbf{u} \\
& \boldsymbol{\beta}_{B}=i \frac{\mathbf{B}}{\sqrt{\rho \mu}} \\
& \beta_{3}=\frac{1}{k T}
\end{aligned}
$$

Using the above definitions we obtain the local complex Boltzmann Maxwellian distribution function of charged particles with drift velocity $\mathbf{u}$ including the electric field contribution and statistical term including the $\psi$ as thermal velocity and statistical term as a 
function of $\mathbf{B}$, taking in to account the axial behavior of magnetic field.

$$
f_{M}=\frac{\rho m^{1 / 2}}{(2 \pi k T)^{3 / 2}} \exp \left\{-\frac{m}{2 k T}\left|(\xi-\mathbf{u})-i \frac{\mathbf{B}}{\sqrt{\mu \rho}}\right|^{2}\right\},
$$

where:

$\rho$ is the density,

$\boldsymbol{\xi}$ is the particle velocity

$\mathbf{u}$ is the drift velocity,

$T$ includes also the electromagnetic contribution,

$\mu$ is the magnetic permeability, in general is a function of $\rho$.

\subsection{The Macroscopic Magneto Gas Dynamic Parameters}

The macroscopic hydrodynamic and electromagnetic observables are obtained respectively as the real and imaginary part of the integral along the line $L$ parallel to the real axis and shifted by $i \mathbf{B} / \sqrt{\mu \rho}$ in the imaginary axis direction.

$$
\begin{gathered}
\rho=\int_{L} m f_{M} d^{3} \boldsymbol{\xi} \\
\mathbf{u}=\frac{1}{\rho} \int_{L} m \boldsymbol{\xi} f_{M} d^{3} \boldsymbol{\xi} \\
E=\int_{L} \frac{1}{2} m \boldsymbol{\xi}^{2} f_{M} d^{3} \boldsymbol{\xi} \\
\mathbf{B}=-\frac{1}{\sqrt{\mu \rho}} \int_{L} m \boldsymbol{\xi}^{*} f_{M} d^{3} \boldsymbol{\xi}
\end{gathered}
$$

Thus, by using this distribution function to calculate the mass, momentum and energy fluxes most of the electromagnetic contribution is calculated directly, i.e. one does not have to solve the hydrodynamic and magnetic force components separately or differently, as it will be shown below.

\section{RECONSTRUCTION OF THE IDEAL MAGNETO GAS DYNAMICS SYSTEM OF EQUATIONS}

In order to provide the validity of the proposed statistical complex distribution function, we show that the equilibrium condition reproduces correctly the ideal magneto gas dynamics system of equations. 
The magneto gas dynamics system of equations is obtained by the integration of Eq. (1) with vanishing collision integral along the line $L$ with respect to the particle velocity $\boldsymbol{\xi}$. The summational invariants $\phi(\xi)=\left(m, m \boldsymbol{\xi}, \frac{1}{2} m \boldsymbol{\xi}^{2}\right)$ and $\phi(\xi)=m \boldsymbol{\xi}^{*}$ are used, respectively, for the gas dynamics observables and the magnetic induction:

$$
\begin{aligned}
& \int_{L} \phi(\boldsymbol{\xi}) \frac{\partial}{\partial t} f_{M}(\mathbf{x}, \boldsymbol{\xi}, t) d \boldsymbol{\xi} \\
& +\int_{L} \phi(\boldsymbol{\xi}) \operatorname{div}\left(\boldsymbol{\xi} f_{M}(\mathbf{x}, \boldsymbol{\xi}, t)\right) d \boldsymbol{\xi}=0
\end{aligned}
$$

The continuity equation for the density is obtained from the real part of Eq. (41) with the summational invariant $\phi(\boldsymbol{\xi})=m$ :

$$
\begin{aligned}
\Re: & \int_{L} m \frac{\partial}{\partial t} f_{M}(\mathbf{x}, \boldsymbol{\xi}, t) d \boldsymbol{\xi} \\
& +\int_{L} m \frac{\partial}{\partial x_{i}}\left(\xi_{i} f_{M}(\mathbf{x}, \boldsymbol{\xi}, t)\right) d \boldsymbol{\xi}=0 ;
\end{aligned}
$$

After substituting the integration variable and inserting the proposed distribution function, we obtain:

$$
\begin{aligned}
& \frac{\partial}{\partial t} \int_{-\infty}^{+\infty} m \frac{\rho m^{1 / 2}}{(2 \pi k T)^{3 / 2}} \exp \left\{-\frac{m}{2 k T}\left|\boldsymbol{\xi}^{\prime}\right|^{2}\right\} d \boldsymbol{\xi}^{\prime} \\
& +\frac{\partial}{\partial x_{i}} \int_{-\infty}^{+\infty} m\left(\xi_{i}^{\prime}+u_{i}\right) \frac{\rho m^{1 / 2}}{(2 \pi k T)^{3 / 2}} \exp \left\{-\frac{m}{2 k T}\left|\boldsymbol{\xi}^{\prime}\right|^{2}\right\} d \boldsymbol{\xi}^{\prime} \\
& =0
\end{aligned}
$$

Finally from the evaluation of the integrals in Eq. (43) we obtain:

$$
\frac{\partial \rho}{\partial t}+\frac{\partial \rho u_{i}}{\partial x_{i}}=0
$$

Similarly, using the summational invariants $\phi(\boldsymbol{\xi})=\left(m \boldsymbol{\xi}, \frac{1}{2} m \xi^{2}\right)$ respectively, we obtain from (41) the conservation laws for the momentum and energy, including the magnetic field contribution:

$$
\begin{aligned}
& \frac{\partial}{\partial t} \rho u_{i}+\frac{\partial}{\partial x_{k}}\left[\left(p+\frac{B^{2}}{2 \mu}\right) \delta_{i k}+\rho u_{i} u_{k}-\frac{B_{i} B_{k}}{\mu}\right]=0 \\
& \frac{\partial}{\partial t} E+\frac{\partial}{\partial x_{i}}\left[u_{i}\left(E+p+\frac{B^{2}}{2 \mu}\right)-\frac{B_{i}}{\mu} \sum_{k} u_{k} B_{k}\right]=0
\end{aligned}
$$


The total energy density includes thermal, kinetic and magnetic energies:

$$
E=\frac{3}{2} p+\frac{1}{2} \rho u^{2}+\frac{B^{2}}{2 \mu}
$$

The magnetic induction evolution equation is obtained from the imaginary part of Eq. (41) with the summation invariant $\phi(\boldsymbol{\xi})=\left(m \boldsymbol{\xi}^{*}\right)$ :

$$
\begin{aligned}
& \Im: \int_{L} m \xi_{i}^{*} \frac{\partial}{\partial t} f_{M}(\mathbf{x}, \boldsymbol{\xi}, t) d \boldsymbol{\xi}+\int_{L} m \xi_{i}^{*} \frac{\partial}{\partial x_{k}}\left(\xi_{k} f_{M}(\mathbf{x}, \boldsymbol{\xi}, t)\right) d \boldsymbol{\xi} \\
& \quad=0 ;
\end{aligned}
$$

After substituting the integration variable and inserting the distribution function, we get the equation for the imaginary part as:

$$
\begin{aligned}
& -\frac{\partial}{\partial t} \int_{-\infty}^{+\infty} m \frac{B_{i}}{\sqrt{\mu \rho}} \frac{\rho m^{1 / 2}}{(2 \pi k T)^{3 / 2}} \exp \left\{-\frac{m}{2 k T}\left|\boldsymbol{\xi}^{\prime}\right|^{2}\right\} d \boldsymbol{\xi}^{\prime} \\
& -\frac{\partial}{\partial x_{k}} \int_{-\infty}^{+\infty}\left(m \frac{1}{\sqrt{\mu \rho}}\left(\xi_{k}^{\prime} B_{i}-\xi_{i}^{\prime} B_{k}+u_{k} B_{i}-u_{i} B_{k}\right)\right. \\
& \left.\frac{\rho m^{1 / 2}}{(2 \pi k T)^{3 / 2}} \exp \left\{-\frac{m}{2 k T}\left|\boldsymbol{\xi}^{\prime}\right|^{2}\right\}\right) d \boldsymbol{\xi}^{\prime}=0
\end{aligned}
$$

The evaluation of the Eq. 49 gives:

$$
\frac{\partial}{\partial t} B_{i}+\frac{\partial}{\partial x_{k}}\left(u_{k} B_{i}-u_{i} B_{k}\right)=0
$$

For the compressible media, we use the linear approximation of the magnetic permeability:

$$
\mu=\frac{\mu_{v}}{\rho}
$$

where $\mu_{v}$ is a constant representing the magnetic

permeability per volume.

\section{CONCLUSIONS}

We propose the novel complex Boltzmann Maxwellian distribution function which includes electromagnetic interactions in a natural way and gives the possibility to reconstruct the macroscopic observables and magneto gas dynamic equations through moments of the distribution function. 
This approach gives the possibility to use the proposed distribution function for the solution of the magneto gas dynamic problems by kinetic consistent methods and lattice Boltzmann methods, which are now considered as the most perspective ones for the calculation on modern parallel computing systems [15] .

The computational kinetic schemes with the use of the proposed complex distribution function provide a methods derive the full magneto gas dynamic equations for the charged particles in a more physical way.

\section{ACNOWLEDGEMENT}

This study was supported by Russian Science Foundation grant 14-11-00170

\section{REFERENCES}

[1] Boltzmann L.,Vorlesungen uber Gastheorie, part I, 1896, part II, 1898, "Lectures on Gas Theory" (translation), University Of California press, 1964.

[2] Succi S., The Lattice Boltzmann Equation, Claredon Press, Oxford press, 2001.

[3] Chetverushkin B., Elizarova T., USSR Comput. Math.Phys, 25 (1985) 164.

[4] Batchelor G., An Introduction to Fluid Dynamics, Cambridge University Press,1967.

[5] Vlasov A., J. Experimental and Theoretical Physics 8 (1938) 291.

[6] Croisille J.-P., Khnfir R. and Chanteur G., J. Sci. Comput.10 (1995) 81.

[7] Huba J.D. and Lyon J.G., J. Sci. Comput. 61 (1999) 391.

[8] Dellar P.J., J. Comput. Phys.179 (2002) 391.

[9] Chetverushkin B., Russ. J. Numer. Anal. Math. Modelling, 20 (2005) 337.

[10] Chetverushkin B., Kinetic Schemes and Quasi-Gas Dynamic System of Equations, CIMNE, 2008.

[11] Tones L. and Allis W.P., Phys. Rev. 52 (1937) 117.

[12] Graebel W.P., Advanced Fluid Mechanics, Academic Press, Elsevier (2007).

[13] L.Landau L. and Lifshitz L., The Classical Teory of Fields, Volume 2 of a Course of Theoretical Physics, Pergamon Press, 1971.

[14] L.Landau L. and Lifshitz L., The Physics Kinetic , Volume 10 of a Course of Theoretical Physics, Pergamon Press, 1971.

[15] Ahern S. et al., ORNL/TM-2007/238 (2007). 